\title{
Infrecuente cáncer no melanoma: carcinoma mucinoso
}

\author{
Infrequent non-melanoma cancer: Cutaneous mucinous carcinoma
}

\section{Manuela Duque-Ruiz' ${ }^{1}$ Elsa Vásquez-Trespalacios ${ }^{2}$}

1. Médica, Facultad de Medicina, Universidad CES, Medellín, Colombia

2. Bióloga, magíster en Epidemiología; investigadora de posgrados clínicos, Facultad de Medicina, Universidad CES, Medellín, Colombia.

\section{RESUMEN}

El carcinoma mucinoso es una entidad rara y maligna de las glándulas ecrinas. Fue descrito en 1952 y se caracteriza por la producción de mucina en los islotes del tumor; es de crecimiento lento y se presenta como nódulos asintomáticos ubicados en la cabeza, el cuello, alrededor de los párpados y en el cuero cabelludo. Puede reaparecer con el tiempo. El tratamiento de elección es la resección quirúrgica, aunque no se dispone de un estándar de tratamiento quirúrgico, frecuentemente se utilizan la clásica resección con $1 \mathrm{~cm}$ de margen y la cirugía de Mohs. Se presenta el caso de un hombre con una lesión multilobulada de evolución crónica, localizada en el ángulo ocular externo. Anteriormente, había sido tratada con resección, pero por su recidiva, se practicó una biopsia. En esta se observó el patrón histopatológico característico de la mucina. Se descartó el compromiso metastásico con estudios de extensión que fueron negativos y se practicó cirugía mediante la técnica diferida de Mohs.

PALABRAS CLAVE: neoplasia cutánea, adenocarcinoma, glándulas sudoríparas, adenocarcinoma mucinoso.

\section{SUMMARY}

Mucinous carcinoma is a rare malignant entity of the eccrine glands, first described in 1952. It is a slow growth tumor, characterized by the production of mucin in the tumor islets. It is described as asymptomatic nodules located on the head, neck, around the eyelids and on the scalp that may relapse over time. The treatment of choice is surgical resection. There is no standardized surgical approach but the resection with $1 \mathrm{~cm}$ margins and Mohs surgery are frequently used.

We present the case of a man who presents with a chronic multilobulated lesion in the external ocular angle after being treated with surgical resection, indicating relapse. The patient is diagnosed with a biopsy that shows a histopathologic pattern characteristic of mucin. Work up rules out metastatic involvement and surgical management of deferred Mohs is performed.

KEY WORDS: skin neoplasms, adenocarcinoma, mucinous, sweat glands.

\author{
Correspondencia: \\ Manuela Duque Ruiz \\ Email: \\ eladuqueo9@gmail.com \\ Recibido: 29/06/18 \\ Aceptado: 03/08/18
}

Conflictos de interés:

No se reportan conflictos de interés.

Financiación:

Ninguna. 


\section{CASO CLIINICO}

Se trata de un paciente de sexo masculino de 84 años de edad, procedente de Nariño (Antioquia), con antecedentes de aneurisma de aorta abdominal corregido quirúrgicamente, insuficiencia arterial en miembro inferior derecho, dislipidemia e hipertensión arterial en tratamiento con hidroclorotiacida, losartán, atorvastatina, ácido acetil-salicílico y amlodipino.

Consultó por presentar una lesión periorbitaria derecha, única y asintomática, de 10 años de evolución, la cual había sido resecada por un médico general cinco años antes. La lesión reapareció y alcanzó un mayor tamaño. Inicialmente, acudió al centro de salud de su zona residencial, de donde fue remitido a un nivel de mayor complejidad para el estudio y diagnóstico de la lesión. Allí le tomaron una biopsia que fue concluyente para carcinoma mucinoso, en la cual se observaron islas de células epiteliales flotando en espacios amplios (figura 1), que corresponden a lagos de mucina intensamente positivos con el marcador azul alciano (figura 2). Las células fueron positivas para GATA-3, receptores de estrógenos,

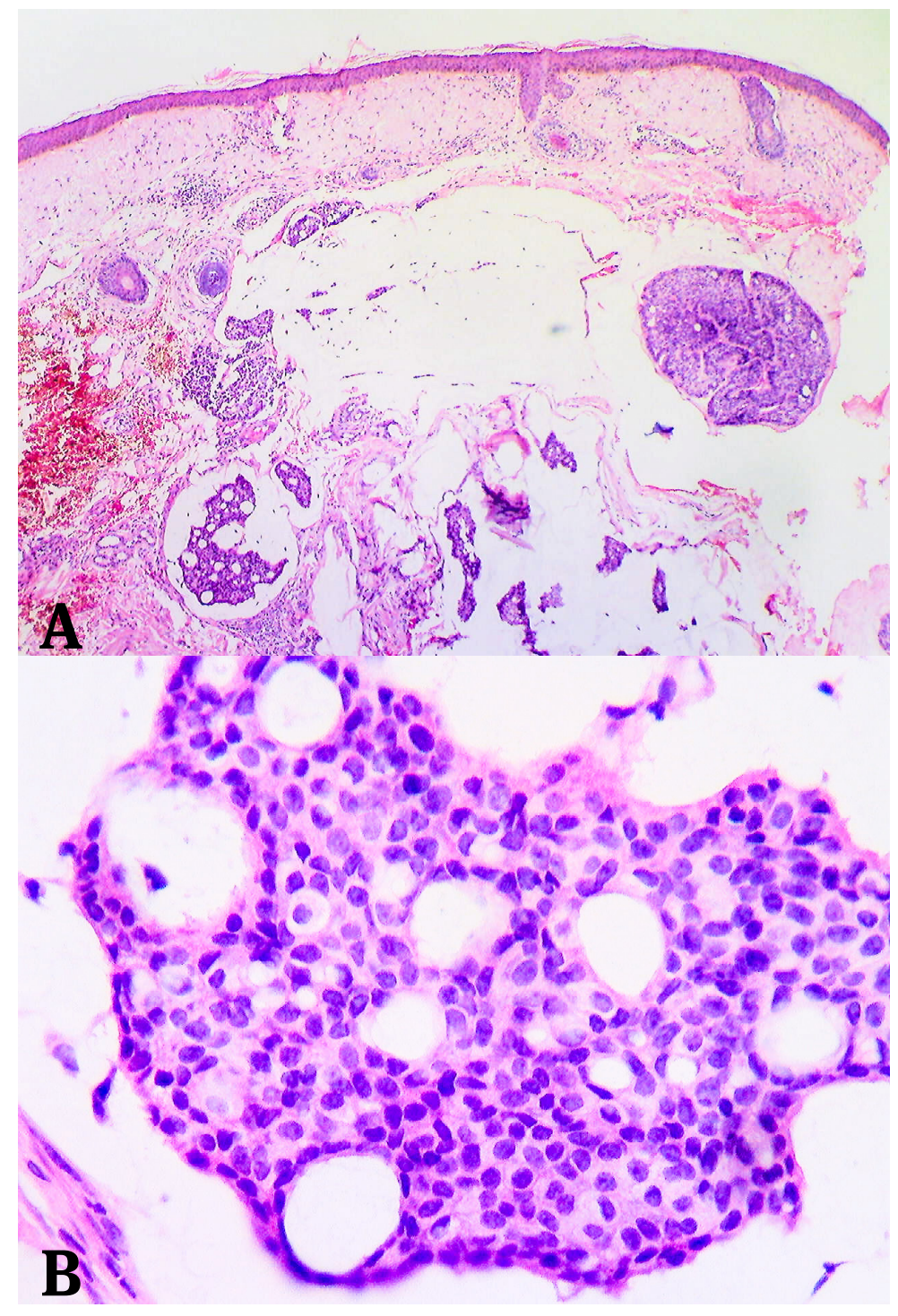

Figura 1. A) Células epiteliales separadas por conglomerados de mucina. Hematoxilina y eosina; 10x B) Acercamiento de nidos de células epiteliales pequeñas ovaladas con escasa atipia y con patrón cribiforme donde se denotan los islotes de mucina en la capa epitelial. Hematoxilina y eosina, 20x. 


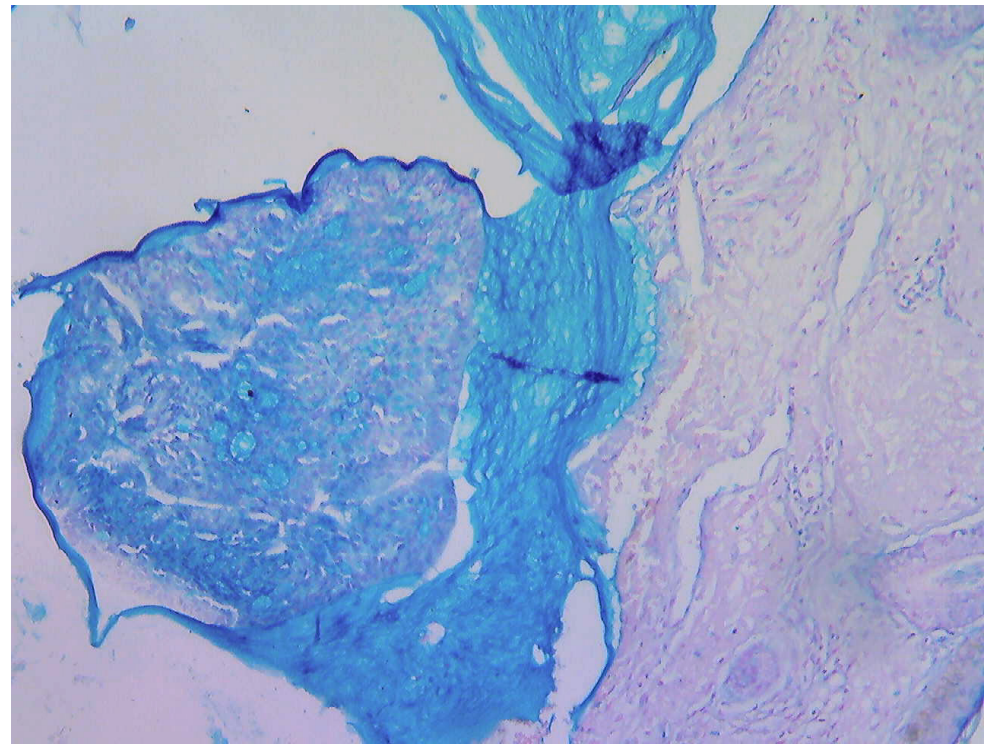

Figura 2. Lagos de mucina entre el tejido con tinción positiva con el marcador azul alciano, 10X.

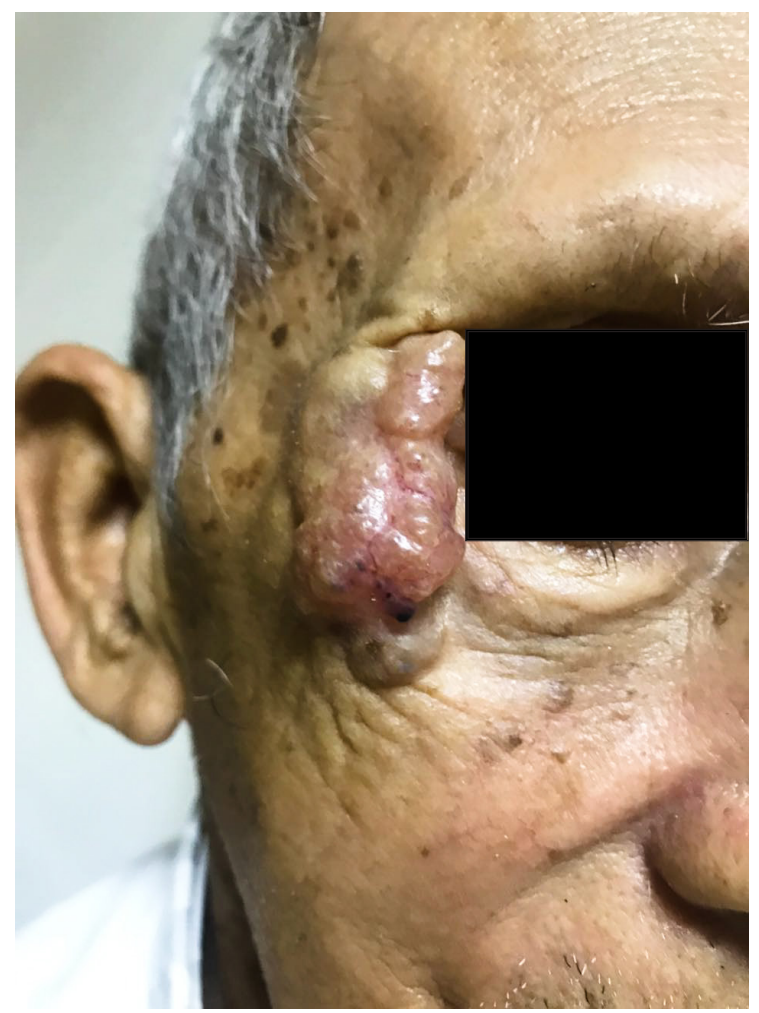

Figura 3. Lesión tumoral peritemporal, multinodular, brillante, eritematosa, móvil e indolora con engrosamiento de la epidermis. receptores de andrógenos, coctel de citoqueratinas y anticuerpos EMA (IgA anti-endomysial antibodies). El marcador p63 fue negativo en esta muestra.

La falta de expresión del p63 en la biopsia, hizo recomendable descartar el compromiso cutáneo secundario, por lo cual le practicaron ecografía mamaria, colonoscopia, endoscopia y tomografía axial toraco-abdominal, que no informaron hallazgos patológicos sugerentes de malignidad o de extensión de la enfermedad; tampoco había compromiso ganglionar y no hubo necesidad de otros exámenes de extensión, como el antígeno prostático específico (PSA).

En el examen físico, la piel era de fototipo III y se apreciaba una lesión multilobulada de apariencia traslúcida, eritematosa, móvil y de consistencia dura, de 4 x 2,5 cm. Estaba localizada en la zona témporo-orbitaria derecha, y se extendía desde la cola de las cejas hasta la región malar; comprometía el borde externo del párpado superior e inferior, aparentemente con bordes infiltrados con múltiples telangiestasias superficiales. Alrededor de la lesión, el paciente presentaba fotodaño moderado (figura 3). Por lo demás, se encontraba asintomático, y negó haber perdido peso o presentar otras lesiones; no se palparon masas ni adenopatías en el cuello o la cabeza.

Por este cuadro clínico, se decidió hacer los estudios de extensión mencionados anteriormente, además resecar la lesión mediante la cirugía diferida de Mohs. No se pre- 
sentaron complicaciones en la intervención quirúrgica. En un primer tiempo, se logró la resección total de la lesión. Posteriormente, se requirió una reconstrucción de cirugía plástica por un lagoftalmos lateral de $2 \mathrm{~mm}$ debido a tracción excesiva del párpado superior.

Finalmente, se hizo un tratamiento localizado, sin que se requirieran otros procedimientos, como vaciamiento ganglionar, quimioterapia o radioterapia coadyuvantes en caso de metástasis secundarias, y no hubo reincidencia de la enfermedad.

\section{DISCUSIÓN}

El carcinoma mucinoso primario cutáneo es un cáncer infrecuente de piel ${ }^{(1-3)}$, lo cual se demuestra con los 200 casos, aproximadamente, publicados en la literatura científica ${ }^{(2,4)}$. En estos casos, fue más frecuente en hombres que en mujeres con una proporción de 2 a 1 (1) con una edad de presentación entre los 50 y los 70 años de edad (media=65 años) ${ }^{(2)}$. Es más frecuente en la raza blanca, lo que hace pensar que el daño por la luz ultravioleta puede ser un factor de riesgo para presentar este carcinoma; además, se presenta en zonas expuestas a la luz. Su localización más común es alrededor de los párpados (49,7\%), seguida por el cuello $(19,5 \%)$ y, por último, la cabeza y el cuero cabelludo (17 $\%)^{(2,5)}$, pero puede presentarse en otras zonas.

Este carcinoma es similar morfológica e histológicamente a los de pulmón, ovario y próstata, al carcinoma in situ ductal endocrino de la mama y al carcinoma papilar sólido mamario ${ }^{(2,6)}$. Por esta razón, se debe descartar la presencia de metástasis en pacientes con lesiones correspondientes a carcinoma mucinoso en estas localizaciones, las que podrían diferenciarse histológicamente por la abundante producción de mucina. Otros diagnósticos diferenciales que se deben considerar son: quiste epidermoide, hemangioma, chalazión, carcinoma basocelular, carcinoma escamocelular, melanoma, carcinoma sebáceo y, el más importante, la metástasis cutánea de un adenocarcinoma primario $^{(4)}$.

En la identificación y en la diferenciación de estos tumores con respecto a otros, se cuenta con la ayuda histológica y patológica de la inmunohistoquímica con tinciones como la de hematoxilina y eosina, en las cuales se pueden observar los conglomerados de mucina, su característica principal, con cordones o nidos de células epiteliales pequeñas, ovaladas, con escasa atipia y con patrón cribiforme (figura 1). La mucina se tiñe con azul alciano (figura 2), PAS y hierro coloidal. Los receptores de estrógenos y progesterona pueden ser débilmente positivos, lo que los distingue de otros carcinomas. Además, las células tumorales expresan la proteína $\mathrm{S} 100$ y el antígeno carcinoembrionario ${ }^{(2,7,8)}$. El paciente puede estar asintomático o presentar síntomas relacionados con cáncer, como pérdida de peso, adenopatías, astenia, etc., acompañados por una lesión multilobulada, eritematosa, translúcida, que altera la estructura en donde se presenta.

$\mathrm{Su}$ diagnóstico puede ser un tanto complejo clínicamente, por su presentación inusual que conlleva subestimar su diagnóstico y recibir un tratamiento inadecuado, además de que no hay un tratamiento fiable por su escasa presentación. En estos casos, se ha visto que la resección clásica del tumor respetando $1 \mathrm{~cm}$ de margen de la lesión, logra disminuir la posibilidad de que la lesión reaparezca.

En algunos estudios publicados, se encontró que los pacientes tratados con la cirugía de Mohs podrían tener mayor posibilidad de recaída, ya que la dificultad para interpretar las secciones congeladas de tejido puede contribuir a algunas fallas de este tratamiento ${ }^{(1)}$. No obstante, se concluyó lo contrario en un meta-análisis publicado en JAMA ${ }^{(5)}$, en el cual se evaluó la evolución de 159 pacientes después del tratamiento quirúrgico con cirugía clásica o con la de Mohs, con poblaciones comparativas inequitativas pero concluyentes; el 9,4\% de los casos fueron tratados con cirugía de Mohs y, el $85,5 \%$, con la cirugía tradicional. De los 15 casos tratados con la cirugía de Mohs, solamente 2 (13\%) presentaron recaídas y no hubo ninguno con metástasis; de los 136 pacientes tratados con cirugía tradicional por escisión, en 46 (34\%) hubo recurrencia o se presentaron metástasis. Esto sugiere que es más favorable el tratamiento con cirugía de Mohs por la resección amplia que permite un completo control de las márgenes, por el reporte de menos casos de metástasis y recurrencia, por los resultados estéticos y cosméticos ${ }^{(5,6,9)}$. La quimioterapia y la radioterapia se utilizan poco en estos tumores ${ }^{(2)}$ ya que a veces basta con su extirpación y, además, se ha demostrado que, en el cáncer mucinoso que tiende a recurrir, la reacción a esta terapia es pobre $^{(1)}$.

Asimismo, en un estudio sobre el pronóstico de este tipo de cáncer, Kamalpour, et al. 10, concluyeron que los factores de riesgo para el pronóstico, la recurrencia y las metástasis, son la localización de las lesiones en el tronco y su aparición a una edad temprana, con un seguimiento a largo plazo de la enfermedad, factores que se asocian con un mal pronóstico. Por otra parte, el pertenecer a la población asiática y el ser tratados con la cirugía de Mohs se asocian con disminución de la recurrencia y menos casos de metástasis y, por lo tanto, con un mejor pronóstico ${ }^{(8)}$. 


\section{CONCLUSIÓN}

Se presenta el caso de un adulto mayor de sexo masculino, con un carcinoma mucinoso, en quien se buscó confirmar inicialmente el diagnóstico por medio del estudio histopatológico, ya que se puede incurrir en un error diagnóstico por la similitud de la clínica y la histología con las de otros carcinomas; el carcinoma mucinoso se diferencia por la presencia característica de abundantes depósitos de mucina, y de receptores de estrógenos y progestágenos.

Además, por la presentación inusual de estas lesiones, se puede desconocer el tratamiento y el seguimiento adecuados de esta enfermedad, por lo cual se hace énfasis en este caso clínico, y se describe como beneficiosa la cirugía de Mohs para su extirpación, con un mejor pronóstico a largo plazo con respecto a la reincidencia y a las metástasis, que deben descartarse desde un principio.

\section{REFERENCIAS}

1. Leal-Guevara LV, Escandón-Martínez ME, Martínez-Pérez L, Fuentes-Sánchez M. Carcinoma mucinoso cutáneo primario. Dermatol Rev Mex. 2015;59:219-22.

2. Mardi K, Diwana VK. Primary cutaneous mucinous carcinoma: A rare entity. Indian Dermatology Online Journal. 2011;2:82-4. doi:10.4103/22295178.85997.

3. Javaid H, Raza N, Ejaz U, Sarfraz T. Unusual skin mass (primary cutaneous mucinous carcinoma). BMJ Case Rep. 2018;2018. pii: bcr-2017-222546. doi: 10.1136/bcr-2017-222546.

4. Stevenson ML, Meehan SA, Vogel L. Primary mucinous carcinoma: A diagnosis of exclusión. J Am Acad Dermatol. 2015;72:AB19o.

5. Kamalpour L, Brindise RT, Nodzenski M, Bach DQ, Veledar E, Alam M. Primary cutaneous mucinous carcinoma systematic review and meta-analysis of outcomes after surgery. JAMA Dermatol. 2014;150:380-4. doi:10.1001/jamadermatol.2013.6006.

6. Emanuel PO, de Vinck D, Waldorf HÁ, Phelps RG. Recurrent endocrine mucin-producing sweat gland carcinoma. Ann Diagn Pathol. 2007;11:44852.

7. Areán-Cuns C, Córdoba-Iturriagagoitia A, AguiarLosada B, Yanguas-Bayona I. Carcinoma mucinoso primario cutáneo. Actas Dermo-Sifiliográficas. 2017;108:884-6.
8. Brinster NK, Liu V, Diwan AH, McKee PH. Prognosis and treatment. Primary cutaneous mucinous carcinoma. High-Yield Pathology. 2011; volumen 1, 434-5.

9. Chavez A, Linos K, Samie FH. Primary cutaneous mucinous carcinoma of the eyelid treated with Mohs surgery. JAAD Case Reports. 2015;1:85-7.

10. Kamalpour L, Bohaty B, West D, Veledar E, Alam M. Prognostic factors for the development of recurrence or metastases in patients with primary cutaneous mucinous carcinoma. JAM Acad Dermatol. 2013;68:AB165. 